\title{
Advances in the practice of electroconvulsive therapy
}

\author{
Toni Lock
}

The first electroconvulsive treatment was administered by Cerletti and Bini in 1938. The event was essentially an experiment, carried out like a military operation (Endler, 1988). The patient was stimulated three times, each time increasing the intensity of the stimulus before a generalised seizure was induced. He had been suffering from an acute psychosis with a poor prognosis, but responded to a course of 11 treatments and was discharged free of symptoms two months later. The first paper on electroconvulsive therapy (ECT) in English was published in the Lancet (Kalinowski) in 1939. At that time, somatic treatment alternatives for the severely ill in large mental institutions included lobotomy and insulin coma therapy. In comparison, unmodified ECT (albeit associated with a significant risk of serious physical morbidity) was predictable, efficient, quick and effective. It is understandable why the treatment became widely and fairly indiscriminately adopted before systematic objective evidence of its efficacy was collected.

The introduction of general anaesthesia and muscle relaxants reduced the risk of physical morbidity, and more recent improvements of anaesthetic procedures and patient monitoring have further improved the safety of ECT. The morbidity rate today ( 1 death per 50000 treatments) is similar to that of general anaesthesia in minor surgical procedures (Kramer, 1985).

That ECT is safe and dramatically effective in some cases is not disputed by the medical profession or the lay public (MIND, 1988). Nevertheless, ECT continues to evoke strong feelings, because of its history of indiscriminate use, and because of an innate fear of the treatment itself - deliberate administration of electric shocks to the brain to induce epileptiform seizures. Given the considerable advances in antipsychotic and antidepressant drug treatment over the last 40 years, does ECT still have a place in contemporary psychiatry?
Research into ECT has focused on efficacy in specific syndromes, indications for treatment, mode of action and side-effects. The focus over the last ten years has been on maximising the therapeutic response while minimising the cognitive side-effects of treatment. In general, ECT research has tended to generate questions faster than it has resolved them. The debate in Britain over the last two years has focused on the advantages and disadvantages of 'stimulus dosing', a concept which is new to many British psychiatrists. The practice, however, is not a recent invention; Cerletti and Bini stimulus dosed their first patient.

This paper provides a broad overview of the recent research and audit findings that have driven changes in British ECT practice since the first ECT audit report to the Royal College of Psychiatrists (Pippard \& Ellam, 1981).

\section{Indications}

Electroconvulsive therapy tends to be reserved as a second-line treatment for patients who have failed to respond to an adequate trial of drugs, in particular antidepressant drugs for depressive illness. The treatment is used less frequently as a first-line intervention for:

(a) patients who are unwilling or unable to tolerate the side-effects of effective drug treatment

(b) patients who in the past responded well to ECT but not to antidepressants

(c) cases where a fast response to treatment is needed to relieve intense suffering or to save the life of the patient, because of either an immediate risk of suicide, or death from other causes (e.g. deep melancholic syndromes,

Dr Toni Lock is Consultant in General Adult Psychiatry and Consultant in Charge of ECT at the Acute Directorate, North Mersey Community (NHS) Trust, Arundel House, Sefton General Hospital, Smithdown Road, Liverpool L15 2LF. She is a member of the Royal College of Psychiatrists' Special Committee on ECT. 
lethal catatonia, and manic frenzy leading to exhaustion).

Empirical clinical and research evidence supports the efficacy of ECT in the treatment of a wide range of psychiatric disorders, including major depressive illness, mania, acute schizophreniform psychoses, catatonic states, neuroleptic malignant syndrome, postpuerperal psychosis, psychiatric disorders associated with epilepsy, spontaneous epileptic disorders per se, idiopathic Parkinson's disease, and drug-induced extrapyramidal disorders. The reader is referred to The Practical Administration of Electroconvulsive Therapy (Royal College of Psychiatrists, $1989,1994)$ and to the equivalent publication of the American Psychiatric Association (1990) for reviews of research data in relation to specific syndromes.

There is remarkable consistency in the findings of open and controlled clinical trials which show the efficacy of ECT in depressive illness, mania and acute schizophreniform syndromes. Patients treated with ECT only or a combination of ECT and drugs (ECT + antidepressants for depressive illness; ECT + lithium for mania; ECT + neuroleptics for schizophrenia) show a more rapid and complete response in the short term (i.e. the initial 4-6 weeks of treatment) than patients treated only with drugs or no specific drug therapy. ECT is particularly effective for severe depressive illness associated with psychotic delusions (Buchan et al, 1992). ECT is also effective in drug-resistant depression (Prudic et al, 1990) and schizophrenia (Taylor, 1990).

Most, if not all, ECT trials are, however, open to criticism on methodological grounds, which diminishes the weight that can be attached to the conclusions reached (Crow \& Johnstone, 1986), and which contributes to ongoing doubts about its efficacy. The issue not addressed by trials is whether ECT contributes a therapeutic effect which may be achieved by other means. For example, where ECT has been shown to be superior to drug treatment in depressive illness, mania and schizophrenia, control subjects may not have been treated with adequate

Box 1. ECT is usually a second-line treatment, but may be a first-line treatment in some cases. Candidates for ECT include those displaying a combination of:

(a) endogenous affective symptoms

(b) acute (florid or type 1) schizophreniform symptoms

(c) marked alterations in psychomotor activity doses of antipsychotic or antidepressant drugs; where ECT has been shown to be particularly effective in depressive illness associated with delusions, control subjects were not treated with an adequate combination of antidepressant and neuroleptic drugs; where ECT has been shown to bring about faster and more complete symptomatic relief from depressive illness, control subjects were not treated with the potent combination of antidepressants and lithium. Another issue which remains uncertain is whether there are any longer-term advantages of ECT over alternative drug treatments after the initial 4-6 weeks of treatment. The evidence is that the advantage of ECT (on its own or in combination with drugs) over drug-only treatment disappears after the initial treatment period.

Research attempts to define specific indicators for a good outcome to ECT have, to some extent, been complicated by the diagnostic process itself. For example, it is sometimes difficult to determine whether acute paranoid delusions are primary (i.e. part of a primary paranoid psychosis) or secondary (i.e. to a severe affective disorder) without the patient's psychiatric history. Paranoid delusions respond well to ECT, regardless of the primary diagnosis (Buchanet al, 1992). Endogenous affective symptoms (e.g. melancholic or manic type) respond well to ECT, regardless of whether primary (i.e. part of an affective disorder) or secondary (i.e. to schizophrenia). Similarly, marked alterations in psychomotor activity respond well to ECT, regardless of the primary diagnosis.

As with drug treatment, a sudden onset and short duration of illness is an indicator of good outcome. In the case of affective disorders, evidence of premorbid personality maladjustment and neurosis (e.g. hypochondriasis, somatisation and obsessivecompulsive symptoms) are indicative of a poor long-term prognosis, even if there is some shortterm response to ECT.

The decision to offer ECT must rest with the practitioner and should be made after carefully weighing the comparative risks of ECT with those of drug treatments. As ECT is effective in drugresistant depression and schizophrenia then - with hindsight - it is evident that a decision to withhold treatment in the first instance may mean that some patients and their carers may have to endure a more lengthy period of stress and hardship.

\section{Clinical standards}

The first ECT audit report to the Royal College of Psychiatrists (Pippard \& Ellam, 1981) asserted that about one in three ECT clinics was ill-equipped, the 
staff poorly trained, and the treatment ineffective. Considerable improvements have since been made (Pippard, 1992a,b), less so, however, in those aspects of treatment which are the direct responsibility of psychiatrists than those which are not (e.g. anaesthetic and nursing practices). Of particular concern was the finding that approximately one in four treatment applications was unlikely to result in therapeutically effective seizures. Furthermore, the standard of training and supervision of junior doctors was generally below that recommended by the Royal College of Psychiatrists $(1977,1989)$, and most juniors did not use the ECT equipment competently. Some anaesthetists have a very low regard for disinterested, poorly skilled and poorly supervised trainees, whom they describe as 'button pushers' (Haddad \& Benbow, 1994). ECT equipment was another cause for concern.

As a result of these findings the Special Committee on ECT of the Royal College of Psychiatrists was re-established and given a wide remit (Freeman, 1992). Over the last two years, College guidelines have been revised, and the revised edition of The Practical Administration of ECT is in press. About 250 psychiatrists have attended the ECT revision courses run by the Special Committee on ECT as part of the College's efforts towards continuing professional development. A short-list of four approved models of ECT machine has been drawn up on the basis of a review of available ECT machines (Lock, 1994b). A video teaching pack has been produced by the Royal College of Psychiatrists' Special Committee on ECT (Gearney, 1993; Lock, 1994a).

Will standards of practice improve over the next five years? This will depend on the willingness of those who prescribe ECT and those who are responsible for its administration to embrace recommendations for change.

Most of the 'changes' advocated in the official training video are relatively minor, and are based on personal experience, recent research findings, and common sense. Doubts have, however, been expressed about the wisdom of some of these changes (Gearney, 1993); for example, why is it necessary for a nurse to trigger the stimulus from the machine itself rather than from the remote control switch on the electrodes? Part of the answer is given in the training video: even experienced ECT administrators lose concentration and wobble the electrodes; this may result in poor electrical contact, high impedance, a compensatory rise in voltage (automatically performed by the machine) electrical arcing, and skin burns. This risk is higher with British machines, as American machines prevent the operator from stimulating the patient if impedance values are above the safe range (Lock, 1994b).
Teaching trainees how to monitor seizures competently is another small change with potentially important effects. Other recommended modifications of existing practice are more costly in terms of time and money: upgrading ECT equipment, and better training and supervision of junior doctors. It is widely recognised that many consultants in charge of ECT clinics do not take their training and supervision responsibilities sufficiently seriously (Pippard \& Ellam, 1981; Pippard, 1992a,b; Castle et al, 1994). The biggest change is recommendations for treatment schedules with particular reference to stimulus dosing and the related technique of dose titration (see below).

\section{Mode of action of ECT}

Exactly how or why ECT is effective in such a wide range of psychiatric disorders remains unclear. Studies on animals using experimental paradigms mimicking a clinical course of treatment provide evidence of neurotransmitter receptor changes (upregulation and down-regulation), which are thought to underlie the therapeutic effects of ECT and cognitive side-effects ( Lever et al, 1986; Green \& Nutt, 1987). A similar pattern of receptor alterations is seen with antidepressant drugs, and affects serotonin and noradrenalin receptors in particular.

Why, then, is ECT effective in the treatment of acute mania and schizophrenia where, according to the dopamine hypothesis for the mode of action of neuroleptic drugs, disturbed dopamine function would be assumed? ECT also appears to have an effect on brain regions (e.g. nucleus accumbens and substantia nigra) which are associated with disturbed dopamine function in acute psychosis (Lock \& McCulloch, 1991). Thus, if ECT simultaneously exerts an antidepressant and antipsychotic effect through its action on noradrenalin, serotonin and dopamine function, its efficacy in patients manifesting a mixture of endogenous affective and acute psychotic symptoms would be explained in biological terms.

It has long been generally accepted that the induced convulsion is essential for the therapeutic effect of ECT, and that unwanted cognitive sideeffects are related to the amount of electricity used to induce that convulsion (Ottosson, 1960). Surprisingly, studies comparing simulated ('sham') ECT ${ }^{1}$

1. The term sham ECT is used to describe an ECT procedure from which the electrical shock (and hence the induced convulsion) is deliberately omitted for research purposes. Sham subjects are given a general anaesthetic and muscle relaxant as per usual practice. 
with real ECT for depressive illness have shown only small, albeit significant, differences between groups (Crow \& Johnstone, 1986), and have failed to provide unequivocal evidence that the convulsion is the critical element of the treatment response; neither have these studies provided evidence that the convulsion is not important.

Sham ECT is a powerful antidepressant treatment, although not as powerful as real ECT. The word 'placebo' generally refers to an inert or harmless substance or treatment which nevertheless exerts a powerful therapeutic effect because of the patient's belief in them. A course of general anaesthetics is not a placebo intervention and it is therefore incorrect to conclude that ECT has a powerful 'placebo effect'; instead, a strong case can be made for the therapeutic efficacy of repeated general anaesthesia for depressive illness (Freeman, 1994).

There is no objective evidence that ECT causes brain damage (Abrams, 1992). Prospective quantitative magnetic resonance imaging of the brain has found no evidence of structural brain damage after repeated courses of ECT (Scott et al, 1991).

\section{Patient preparation}

A full explanation of ECT in language that patients and their families can understand is important to maintain good working relationships. It has been shown, however, that about one in three patients has "no idea" what treatment entails (Freeman \& Kendell, 1986). Adequate physical preparation comprising at least a recent physical examination and blood tests - is essential for safety reasons. Nevertheless, anaesthetists continue to encounter physically ill patients arriving inadequately prepared for treatment (Haddad \& Benbow, 1994).

The task of obtaining consent is often delegated to junior medical staff, but it is the responsibility of the patient's responsible medical officer (RMO) to ensure that ECT is administered legally , in particular to determine whether there are grounds for compulsory treatment under the Mental Health Act where patients refuse treatment or - by virtue of their mental disturbance - are unable to give 'real' consent to treatment (Pippard \& Taylor, 1994). Real consent assumes that the patient has made a valid decision to agree to treatment - that is, that a full explanation of ECT has been given and that the patient is capable of making a decision based on an understanding of the information. It is generally advisable to regard a patient as not consenting to treatment if real consent is in any way questionable. ECT may only be given without a patient's consent in two circumstances: (a) where urgent action is necessary to save the patient's life, or to prevent serious and immediate danger to self or other people

(b) when a patient is detained under an order which permits compulsory treatment for 28 days or longer and where a doctor appointed by the Mental Health Act Commission certifies that ECT is necessary.

The nature and extent of the information which should be given is summarised in ECT: A Factsheet for You and Your Family (available from the Public Education Department of the Royal College of Psychiatrists). A verbal explanation should be supplemented with written or audio-visual material.

Patients giving real consent are required to sign a consent form in the presence of a doctor who countersigns, as evidence that the correct procedure was undertaken. Failure to undertake the above procedure correctly could constitute legal liability on grounds of negligence, and treatment without consent could constitute battery. Consent is best regarded as an on-going process, for it can be withdrawn at any time, and ECT clinic staff are advised to be alert for the possibility that consenting patients may change their mind at the last minute.

\section{Treatment schedules}

A treatment schedule needs to encompass all the factors known to have an effect on clinical outcome (Shapira et al, 1991a,b). Flexibility is important with respect to maximising the therapeutic response and minimising cognitive side-effects. The main factors to consider are the type of ECT machine, electrode placement, seizure threshold, stimulus dose, and frequency of treatments.

\section{ECT machines}

Most ECT machines manufactured after 1981 deliver electrical stimulation in the form of brief (1-2 ms) square-wave pulses (Fig. 1). 'Brief-pulse'stimulation delivers only a fraction of the electrical energy of older ECT machines (which generated alternating sine-wave current) and it is highly efficient in the induction of seizures (Abrams, 1992). Brief-pulse current is also associated with less memory impairment than sine-wave ECT (Weiner et al,1986). Modern machines estimate the impedance of the patient's head and can be pre-set to deliver a fixed amount of charge. Such machines are termed constant-current apparatus, as they maintain current at a constant value by automatically adjusting 

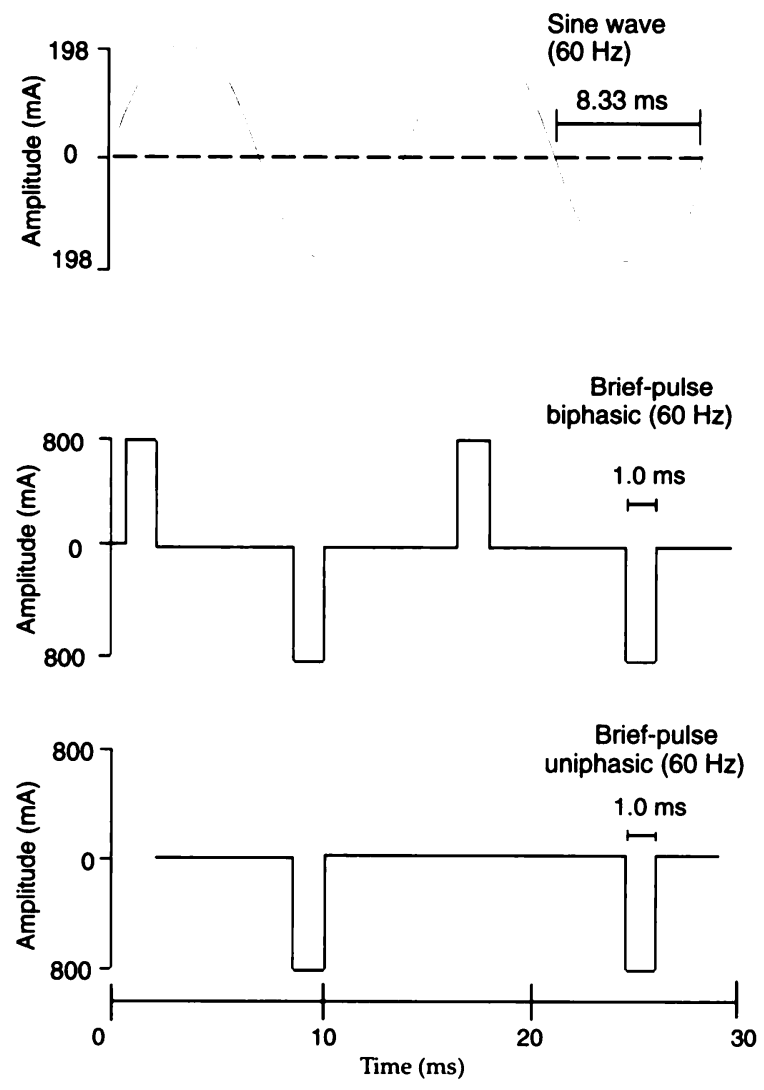

Sine-wave (alternating current; constant voltage), and biphasic and uniphasic brief pulse (constant current) (from Lock, 1994a).

\section{Fig. 1 Wave forms}

output voltage to compensate for changes in impedance, and their use was recommended by the Department of Health and Social Security in 1982.

About $90 \%$ of ECT machines in use in Britain in 1991 (Pippard, 1992a) were of the brief-pulse, constant-current type: Ectron Series $5(54 \%)$, Series 2 and $3(37 \%)$. The remainder were equipped with earlier Ectron Mark 4 (sine-wave) machines.

Selecting an ECT machine is not an easy matter and it must be stressed that there is little advantage in purchasing new equipment without a commitment to understanding how it operates, and learning how to use it competently. Machines differ with respect to the characteristics of the stimulus generated, the means of controlling that stimulus and other features which may be built in. Some of these are important for safety reasons; some are useful but not essential, and others offer little if any practical advantage, despite claims to the contrary by the manufacturers. The best buy for local use requires a general understanding of the merits and limitations of available equipment - and of local
Box 2. ECT machines

Constant-current ECT machines were recommended by the Department of Health and Social Security in 1982.

All ECT machines with the exception of four shortlisted models (British versions of Mecta's SR2 \& JR2, Thymatron DG and Ectron series 5 a models) must now be considered obsolete and replaced.

The best selection for local use requires a general understanding of the merits and limitations of available equipment and of local ECT practice.

ECT practice. The interested reader is referred to Lock (1994a) for a comprehensive review of available equipment.

\section{Electrode placement}

In 1977, the Royal College of Psychiatrists recommended the use of brief-pulse equipment and unilateral electrode placement. By 1989 it was clear that the therapeutic efficacy of this combination was less than when using bilateral ECT and earlier sinewave models. The cause of the problem was that the early Ectron (Series 2 and Series 3 ) constantcurrent machines were under-powered.

Recent American and Scandinavian research using more powerful brief-pulse machines has shown that therapeutic equivalence may be achieved with unilateral and bilateral electrode placement (Abrams et al, 1991).

Effective unilateral treatment is critically dependent on:

(a) a stimulus dose which is at least 2.5 times the patient's seizure threshold

(b) wide separation of the electrodes.

The 'Lancaster' unilateral electrode position (as demonstrated on the old ECT wallcharts; see Fig. 2a) does not achieve a sufficiently wide electrode separation, and the d'Elia electrode positions are recommended (as demonstrated in the Official Video Teaching Pack of the Royal College of Psychiatrists Special Committee on ECT, 1994; see Fig. 2b). Doubts continue to be expressed about the therapeutic equivalency of unilateral and bilateral electrode placement (Sackeim et al, 1991), and small but statistically significant differences in seizure quality have been observed. 
Flexibility with respect to electrode placement is important. The American Psychiatric Association Task Force on ECT (1990) recommended that the decision to use bilateral or unilateral ECT should be based on a risk-benefit analysis for each patient. Bilateral ECT is recommended if the rate of response is most important (e.g. in the case of urgent treatment); unilateral ECT is recommended if minimising the risk of cognitive side-effects is most important. It may, furthermore, be advisable to switch from one to the other during a course of treatment. For example, if a patient develops troublesome cognitive side-effects with bilateral ECT, switching to unilateral ECT may help; similarly, if a patient fails to improve with unilateral ECT and is not experiencing side-effects, switching to bilateral ECT may help.

Non-dominant (unilateral) ECT is associated with significantly fewer unwanted cognitive problems compared with bilateral ECT; patients reorientate faster, and experience less disturbance of objective

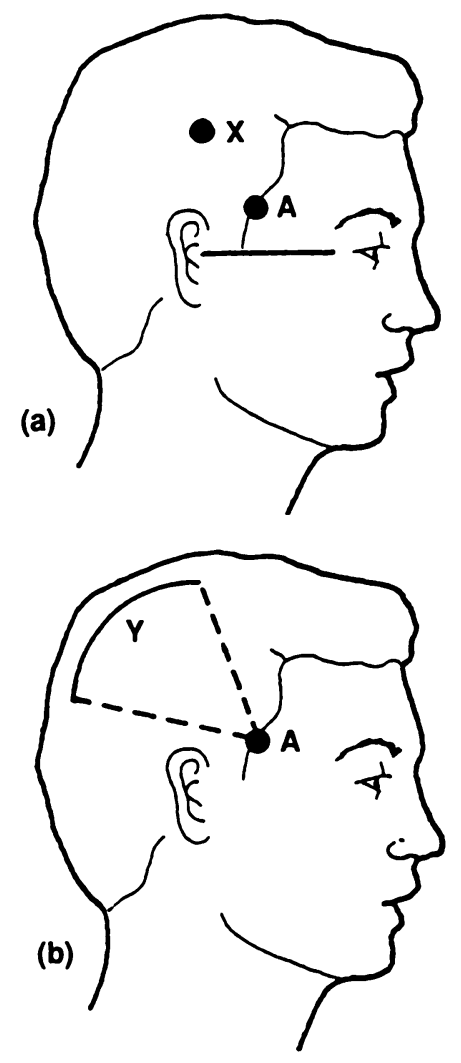

(a) The Lancaster electrode placement positions for unilateral ECT; $A=4 \mathrm{~cm}$ above the midpoint between the external auditory meatus and angle of the orbit; $X=6 \mathrm{~cm}$ from $A$, and above the external auditory meatus. (b) The d'Elia electrode placement positions: $A$, as above; $Y=$ on the midline, anywhere in the region of the occipito-parietal junction.

Fig. 2 Electrode placement positions

\section{Box 3. Treatment schedules}

The decision to use bilateral or unilateral ECT should be based on a risk-benefit analysis. Switch from bilateral to unilateral ECT if a patient develops troublesome cognitive side-effects. Switch from unilateral to bilateral ECT if the patient fails to improve and has no side-effects.

The unilateral use of brief pulse stimulation gives the least cognitive disturbance, while bilateral ECT with sign wave stimulation gives the most.

Combining brief pulse stimulation and bilateral electrode placement offers the best compromise of effective treatment and moderate memory disturbance.

retrograde and anterograde memory impairment, and weaker subjective autobiographical amnesia (Weiner et al, 1986). Electrode placement and stimulus waveform exert synergistic effects on cognitive function; the combination of unilateral ECT and brief-pulse stimulation is associated with the least cognitive disturbance, while the combination of bilateral ECT and sine-wave stimulation gives the most cognitive disturbance. Other combinations offer a compromise of effective treatment and mild memory disturbance (Weiner $e t$ al, 1986).

\section{Seizure threshold and stimulus dosing}

Seizure threshold (ST) refers to the minimum amount of electricity needed to induce a generalised cerebral seizure. The term 'dose' (of electricity) has crept into the literature as an alternative to the term amount.ST may be measured using a variety of units (e.g. volts, V; amps, A; coulombs, Q). The most valid measure is the coulomb, the unit of electrical charge ${ }^{2}$.

The absolute measurement of ST is heavily dependent on the type of ECT machine (Abrams, 1992), but regardless of which machine is used, a wide

2. The amount of charge in millicoulombs, is calculated as follows: multiply current (in amps) by pulse width (in milliseconds) to get the electrical charge of one brief pulse in millicoulombs (mQ); multiply this figure by frequency (the number of pulses per second) and by the length of time that current flows (i.e. the duration of the stimulus in seconds). If the ECT machine produces biphasic brief-pulse current (all American models), this figure must be multiplied by 2 . If the ECT machine produces uniphasic brief-pulse current (e.g. Ectron models) the multiplication factor is 1 . Relevant parameters will be quoted in the manufacturer's instruction manual. (One millicoulomb $(\mathrm{mQ})$ is equal to one-thousandth $(1 / 1000)$ of a coulomb.) 
Table 1. Factors affecting seizure threshold

Factor

Effect

Age (increasing)

Anticonvulsants - concurrent or recent

Baldness

Barbiturates - concurrent or recent

Benzodiazepines - concurrent or recent

Bilateral electrode placement

Bones (thick), e.g. Paget's disease

Caffeine

Carbon dioxide saturation of blood (low)

Dehydration

ECT - increasing no. of treatments

ECT - previous course within last month

Electrode contact with scalp (poor)

Hyperventilation

Methohexitone dose $>1.2 \mathrm{mg} / \mathrm{kg}$

Methohexitone and ketamine in half-doses

Oxygen saturation of blood (low)

Propofol

Sex (male)

Theophyllin

variation in ST has been observed in clinic populations (e.g. 17-800 mQ or more; Sackeim et al, 1987; using a Mecta SR1 machine), and a rise in ST (range $25-200 \%$; mean $80 \%$ ) has been noted during a course of ECT. Factors affecting ST are listed in Table 1. The reader is referred to Sackeim et al $(1987,1991)$ for the relative magnitude of these effects.

'Stimulus dosing' refers to the adjustment of the amount (or dose) of the electrical stimulus to the requirements of the patient at different points during the course of ECT. The consensus of the American Psychiatric Association's Task Force on ECT (1990) is that the best results - in terms of maximising the therapeutic response to ECT while minimising cognitive side-effects - are achieved using a 'moderately suprathreshold treatment dose', that is, a dose which is $50-200 \%$ above ST. The exact percentage increase for bilateral and unilateral ECT is a subject of ongoing international debate: given the controversy, a figure of about $50 \%$ is suggested for bilateral ECT and at least $250 \%$ for unilateral ECT (Sackeim, 1991). In addition, the selected dose may need to be increased during a course of ECT to compensate for the rise in seizure threshold as the course of ECT progresses.

Individualised stimulus dosing using the above guidelines is dependent on knowing the patient's seizure threshold dose. Given the many factors which can, alone or in combination, affect ST, the most reliable assessment of ST is by empirical measurement - a procedure which has been termed 'dose titration', or 'seizure threshold titration'. This is done by applying a low charge stimulus, and restimulating the patient with an increased charge until a seizure is induced. ST titration tables have been developed (see Table 2) for all the short-listed ECT machines, which simplifies the procedure (Lock, 1994c).

The practice of applying a fixed dose to all patients at every treatment session is still common in Britain (Pippard, 1992a). The problem with this approach is that if a fixed high dose is selected, a significant proportion of patients are likely to have relatively low seizure thresholds, and may therefore be at increased risk of cognitive side-effects; with a fixed low-dose schedule, patients with a relatively high ST are at risk of receiving a course of treatment characterised by poor seizures and a poor response.

Whether ST titration should be part of routine clinical practice is a contentious issue. The consensus of the Special Committee on ECT is that ST titration is useful if bilateral ECT is administered, because of the risks to memory function posed by inadvertent gross suprathreshold stimulation; and useful in the case of unilateral ECT because of the need to ensure that a sufficiently high stimulus is given to maximise the therapeutic response. Fixed high-dose schedules are considerably simpler to undertake than the alternative - dose titration - and are appropriate for unilateral ECT (Abrams et al, 1991). Stimulus dosing guidance for bilateral ECT, taking into consideration the three most important factors affecting ST (age, sex and concomitant medication), are given in Table 2, and are based on local experience using a Mecta SR 2 'British version' machine. It

Table 2. Stimulus dosing guidelines for Mecta SR2/JR2 British version machines

Level ST dose: $\mathrm{mQ}$

Treatment dose: $\mathrm{mQ}$ Bilateral Unilateral

$\begin{array}{rrrr}1 & 25 & 50 & 75 \\ 2 & 50 & 75 & 125 \\ 3 & 75 & 125 & 200 \\ 4 & 125 & 200 & 275 \\ 5 & 200 & 275 & 400 \\ 6 & 275 & 400 & 600\end{array}$

Starting levels: 1 = female, unilateral ECT

2 = male, unilateral ECT

$3=$ female, bilateral ECT

$4=$ male, bilateral ECT

Start one level higher if patient is over 65 years old and / or if patient is taking the equivalent of $15 \mathrm{mg} /$ day of diazepam or any anticonvulsant. Increase dose by one level if a stimulation fails to induce a generalised tonic-clonic seizure (maximum 3 stimulations). 
is important to stress that absolute values (in $\mathrm{mQ}$ ) may differ for other models, and users are referred to Lock (1994c) for specific guidance.

\section{Frequency of treatment}

Electroconvulsive therapy is usually administered twice weekly in Britain, and three times weekly in the US, for treatment of depressive illness. This difference appears to be one of personal preference (Scott \& Whalley, 1993). An alternative to switching from bilateral to unilateral electrode placement in cases where cognitive side-effects (in particular confusion) are troublesome is to reduce the frequency of treatments from two to one a week (Benbow, 1994). It has been claimed, on the basis of empirical clinical experience, that daily treatments are indicated when a patient's mental state is seriously disturbed (e.g. uncontrolled manic excitement or catatonia). In the case of continuation (maintenance) ECT, the frequency of treatments should take into consideration the patient's clinical progress. Some patients' symptoms may relapse unless one treatment is administered per week, while others may remain asymptomatic with less frequent treatments.

\section{Monitoring and restimulation}

There are several causes of 'missed fits' (i.e. absence of peripheral seizure activity) and partial seizures (seizure involving only the face, one limb, or both limbs on the same side):

(a) poor electrical contact

(b) total attenuation of muscle activity by muscle relaxant

(c) absence of cerebral seizure activity because the dose of the stimulus was below the patient's seizure threshold, thus failing to induce a generalised cerebral seizure.

Poor electrical contact can easily be excluded by routinely testing that static impedance values are within the recommended range for a given ECT machine before stimulating the patient. The routine use of the cuff technique has been recommended, as it provides the most reliable means of monitoring peripheral seizure activity (Royal College of Psychiatrists, 1989, 1994). This technique makes use of a blood pressure cuff, inflated to about $20 \mathrm{mmHg}$ above systolic blood pressure, which is applied immediately before the muscle relaxant is administered. Muscle relaxant is therefore unable to pass beyond the inflated cuff, where peripheral seizure activity is better observed. This technique is well demonstrated in the official teaching video.

The most direct means of monitoring seizure activity induced by an ECT stimulus is by means of the electroencephalogram (EEG). American ECT machines offer built-in EEG monitoring, which is considered useful but not essential by the Special Committee on ECT of the Royal College of Psychiatrists.

The appropriate action to be taken in response to a missed or partial seizure will depend on its cause. If due to lack of cerebral seizure activity, then the strength of the stimulus needs to be increased; if due to poor electrical contact, then the electrodes should be prepared again and reapplied (and impedance should again be measured). When peripheral seizure activity is absent but cerebral seizure activity is confirmed by means of an EEG recording, then the operator needs to verify that the cerebral convulsion is adequate, and should also liaise with the anaesthetist, as a reduction in the dose of muscle relaxant may be appropriate at subsequent treatment sessions.

A discussion on EEG monitoring is not possible in this paper and the interested reader is referred to Scott et al (1989). Sample EEG recordings are also provided by the Royal College of Psychiatrists (1994). Where built-in EEG recording is not available, the only means of differentiating between inadequate seizures due to absent cerebral activity and total attenuation of peripheral muscular activity by muscle relaxant is by means of the cuff technique.

\section{Conclusions}

This review has attempted to examine the scientific rationale underlying recommended improvements in British ECT practice, in the light of the findings of two audit reports to the Royal College of Psychiatrists (Pippard \& Ellam, 1981; Pippard, 1992a). The nature of ECT is that it is controversial, and research findings are generally inconclusive and open to debate. An attempt has been made throughout this paper to offer explanations based on the consensus opinion of both the Special Committee on ECT of the Royal College of Psychiatrists and the American Psychiatric Association's Task Force on ECT. Where their views differ, the view of the Special Committee has been presented. Both bodies consider ECT to be a safe and effective procedure for which there exists an ongoing need. The caveat is that ECT must be properly and legally admin- 
istered and that the decision to offer or withhold treatment should be based on a careful consideration of alternatives for a given patient, not on the basis of personal bias on the part of the practitioner.

The question we all need to ask ourselves is this: would we, if we were suffering from a severe schizoaffective disorder, consent to ECT treatment in our local ECT clinic?

\section{References}

Abrams, R. (1992) Electroconvulsive Therapy (2nd edn). Oxford: Oxford University Press.

, Swartz, C. M. \& Vedak, C. (1991) Antidepressant effects of high-dose right unilateral electroconvulsive therapy. Archives of General Psychiatry, 48, 746-748.

American Psychiatric Association Task Force on Electroconvulsive Therapy (1990) The Practice of Electroconvulsive Therapy: Recommendations for Treatment, Training and Privileging. Washington, DC: APA. (See also Weiner et al, 1990.)

Benbow, S. (1994) ECT in elderly patients. In The Practical Administration of ECT (2nd edn)(ed.C. P. L. Freeman). London: Royal College of Psychiatrists (in press).

Buchan, H., Johnstone, E., McPherson, K., et al (1992) Who benefits from electroconvulsive therapy? Combined results of the Leicester and Northwich Park trials. British Journal of Psychiatry, 160, 355-359.

Castle, D., Reeve, A., Ivinson, L., et al (1994) What do we think about our training? Report of a Working Party of the Collegiate Trainees' Committee. Psychiatric Bulletin, 18, 357-359.

Crow, T. J. \& Johnstone, E. C. (1986) Controlled trials of electroconvulsive therapy. Annals of the New York Academy of Sciences, 462, 12-29.

Endler, N. S. (1988) The origins of electroconvulsive therapy (ECT). Convulsive Therapy, 4, 5-23.

Freeman, C. P. L. (1992) Special Committee on ECT. Psychiatric Bulletin, 16, 121.

- (1994) Non-convulsive electrical shock treatment. In Practical Administration of ECT (2nd edn). London: Royal College of Psychiatrists (in press).

\& Kendell, R. E. (1986) Patients' experiences of and attitudes to electroconvulsive therapy. Annals of the New York Academy of Science, 462, 341-351.

Gearney, D. (1993) Video tape review of electroconvulsive therapy: the official video of the Royal College of Psychiatrists' Special Committee on ECT. Psychiatric Bulletin, 17, 702-703.

Green, R.A. \& Nutt, D. J. (1987) Psychopharmacology of repeated services: possible relevance to the mechanism of action of electroconvulsive therapy. In Handbook of Psychopharmacology (eds I. L. Iversen, S. D. Iversen \& S. M. Snyder), pp. 375-419. New York: Plenum Press.

Haddad, P. M. \& Benbow, S. M. (1994) Anaesthetists' views of electroconvulsive therapy. Psychiatric Bulletin (in press).

Kalinowski, L. B. (1939) Electroconvulsive therapy in schizophrenia. Lancet, ii, 1232-1233.

Kramer, B. A. (1985) Use of ECT in California, 1977-1983. American Journal of Psychiatry, 142, 1190-1192.

Lever, B., Stanley, M., Altma, M., et al (1986) An annual model of electroconvulsive therapy-induced amnesia. Annals of the New York Academy of Sciences, 462, 91-98.

Lock, T. (1994a) Official Video Teaching Pack of the Royal College of Psychiatrists'Special Committee on ECT. London: Royal College of Psychiatrists.

(1994b) ECT machines. In The Practical Administration of ECT (2nd edn) (ed. C. P. L. Freeman). London: Royal College of Pychiatrists (in press). (1994c) Stimulus dosing. In The Practical Administration of ECT (2nd edn) (ed. C. P. L. Freeman). London: Royal College of Pychiatrists (in press)

_ \& McCulloch, J. (1991) Local cerebral glucose utilization after chronic elecroconvulsive shock: implications for the mode of action of electroconvulsive therapy. Journal of Psychopharmacology, 5, 111-119.

MIND (1988) ECT: Pros, Cons and Consequences (Special Report). London: MIND.

Ottosson, J. O. (ed.) (1960) Experimental studies of the mode of action of electroconvulsive therapy. Acta Psychiatrica Scandinavica (suppl. 145).

Pippard, J. (1992a) Audit of electroconvulsive treatment in two National Health Service regions. British Journal of Psychiatry 160, 621-637.

- $(1992 b)$ Auditing the administration of ECT. Psychiatric Bulletin, 16, 59-62.

— \& Ellam, L. (1981) Electroconvulsive treatment in Great Britain. British Journal of Psychiatry, 139, 563-568.

— \& Taylor, P. J. (1994) ECT, the law and consent to treatment. In The Practical Administration of ECT (2nd edn) (ed. C. P. L. Freeman). London: Royal College of Psychiatrists (in press).

Prudic, J., Sackeim, M. A. \& Devenard, D. P. (1990) Medication resistance and clinical response to electroconvulsive therapy. Psychiatric Research, 31, 287-296.

Royal College of Psychiatrists (1977) Memorandum on the use of electroconvulsive therapy. British Journal of Psychiatry, 131, 261-272.

Royal College of Psychiatrists' ECT Sub-Committee of the Research Committee (1989) The Practical Administration of Electroconvulsive Therapy (ECT). London: Gaskell.

Royal College of Psychiatrists' Special Committee on ECT (1994) The Practical Administration of Electroconvulsive Therapy (ECT) (2nd edn). London: Royal College of Psychiatrists (in press).

Sackeim, H. A. (1991) Optimizing unilateral electroconvulsive therapy. Convulsive Therapy, 7, 201-212.

-, Decina, P., Prohovnik, I., et al (1987) Seizure threshold in electroconvulsive therapy; effects of sex, age, electrode placement, and number of treatments. Archives of General Psychiatry, 44, 355-360.

- Devanand, D. P. \& Prudic, J. (1991) Stimulus intensity seizure threshold and seizure duration: Impact on the efficacy and safety of electroconvulsive therapy. In Psychiatric Clinics of North America: Electroconvulsive Therapy, vol. 14 (4) (ed. C. H. Kellner), pp. 803-843. Philadelphia: Saunders.

Scott, A. I. F., Shearing, P. A. \& Dykes, S. (1989) Would monitoring by electroencephalogram improve the practice of electroconvulsive therapy? British Journal of Psychiatry, 154, 853-857.

-, Turnbull, L. W., Blare, A., et al (1991) Electroconvulsive therapy and brain damage. Lancet, 338, 264.

— \& Whalley, L. J. (1993) The onset and rate of the antidepressant effect of electroconvulsive therapy. British Journal of Psychiatry, 162, 725-732.

Shapira, B., Calev, A. \& Lerer, B. (1991a) Optimal use of electroconvulsive therapy: choosing a tratment schedule. In Psychiatric Clinics of North America: Electroconvulsive Therapy, vol. 14 (4) (ed. C. H. Kellner), pp. 935-946. Philadelphia: Saunders.

_- Hadjez, J., Calev, A., et al (1991b) Schedule of ECT administration: implications for antidepressants efficacy and adverse effects. Biological Psychiatry, 1, 257-259.

Taylor, P. J. (1990) Schizophrenia and ECT - a case for a change in prescription? In Dilemmas and Difficulties in the Management of Psychiatric Patients (eds K. Hawton \& P. Cowan). Oxford: Oxford University Press.

Weiner, R. D., Rogers, H. J., Davidson, J. R. T., et al (1986) Effects of stimulus parameters on cognitive side-effects. Annals of the New York Academy of Science, 462, 315-325.

-, Fink, M., Hammersley, D., et al (1990) The practice of ECT: recommendations for treatment, training and privileging Convulsive Therapy, 6, 85-120. 


\section{Multiple choice questions}

1 One of the following is likely to decrease seizure threshold:
a right unilateral electrode placement
b doses of methohexitone greater than $1.2 \mathrm{mg} /$ $\mathrm{kg}$
c patients taking diazepam
d patients taking carbemazepine

2 A valid reason for ambivalent feelings about ECT is:
a the past history of indiscriminate use
b lack of efficacy
c a high risk of mortality and serious morbidity
d high standards of practice

3 A good outcome to ECT is suggested by:

a personality maladjustment

b a past history of neurotic illness

c coexistent psychomotor alterations, endogenous affective symptoms and florid (Type 1) psychotic symptoms

d old age

4 It is illegal to administer ECT in the following circumstances:

a to patients who consent to treatment without a clear explanation of what the treatment entails

$\mathrm{b}$ to patients giving real consent to treatment who change their minds at the last minute

c to detained patients, with or without real consent d to certain detained patients who are unwilling or unable to give real consent

5 Compared with bilateral ECT given twice a week, maneouvres which may be useful with respect to reducing cognitive side-effects are:

a switching to right unilateral ECT

$b$ reducing the frequency of treatments

c reducing the treatment dose

$\mathrm{d}$ increasing the treatment dose

\begin{tabular}{|llll|}
\hline MCQ answers & & \\
1 & & 4 & \\
a & T & a & T \\
b & F & b & T \\
c & F & c & T \\
d & F & d & F \\
& & & \\
2 & & 5 & \\
a & T & a & T \\
b & F & b & T \\
c & F & c & T \\
d & F & d & F \\
3 & & & \\
a & F & & \\
b & F & & \\
c & T & & \\
d & F & & \\
\hline
\end{tabular}

